\title{
Cerebellar liponeurocytoma
}

INSERM

\section{Source}

INSERM. (1999). Orphanet: an online rare disease and orphan drug data base. Cerebellar liponeurocytoma. ORPHA:251931

Cerebellar liponeurocytoma (cLPN) is a rare slow growing neuronal tumor seen more frequently in females than males, occurring most commonly in the cerebellum but occasionally in the supratentorial compartment or the fourth ventricle and presenting in the 4 th to 6 th decade of life with symptoms of dizziness, headache and gait instability. It often has a high rate of local recurrence. 